\section{ECCOMAS}

Proceedia
UNCECOMP 2017

$2^{\text {nd }}$ ECCOMAS Thematic Conference on Uncertainty Quantification in Computational Sciences and Engineering M. Papadrakakis, V. Papadopoulos, G. Stefanou (eds.) Rhodes Island, Greece, 15-17 June 2017

\title{
RESPONSE OF BEAMS WITH CRACK OF UNCERTAIN-BUT BOUNDED DEPTH SUBJECTED TO DETERMINISTIC OR STOCHASTIC LOADS
}

\author{
G. Muscolino ${ }^{1}$ and R. Santoro ${ }^{1}$ \\ ${ }^{1}$ Department of Engineering, University of Messina \\ Villaggio S.Agata, 98166 Messina Italy \\ e-mail: gmuscolino@unime.it,roberta.santoro@unime.it
}

Keywords: Damaged beam, uncertain crack depth, interval deterministic response, interval stochastic response.

\begin{abstract}
.
Detection of cracks in structural components and identification of their size for structures having beam form is of crucial importance in many engineering applications. For damaged structures the dynamic response changes with respect to the undamaged ones due to the changes produced on their mechanical properties by the presence of the crack.

In this paper the deterministic behavior of a beam with a transverse on edge non-propagating crack is first studied. Moreover the deterministic and stochastic setting pertaining the case in which the crack has an uncertain depth is investigated. Undamaged elements of the beam are modeled by Euler-type finite elements. The uncertain crack depth is modeled as an interval variable and the cracked beam is subjected to both deterministic and zero-mean nonstationary Gaussian random excitations. In the latter case the equation governing the evolution of the main statistics of the response are derived by means of Kronecker algebra.

Once the mathematical model of the beam is defined, the dynamic response is evaluated by applying a numerical procedure based on the philosophy the Improved Interval Analysis via Extra Unitary Interval. In particular the proposed procedure is based on the following main steps: i) to define a finite element model of the beam in which the model of fully open crack is used to represent the damaged element; ii) to model the crack depth as an interval variable; iii) to evaluate in time domain the response for deterministic and stochastic excitation, by adopting an unified approach.
\end{abstract}




\section{INTRODUCTION}

The fracture behavior of structural components subjected to various loading and environmental conditions is of relevance in assessing structural integrity. When a structure is subjected to damage its dynamic response changes due to the change of its mechanical characteristics $[1,2]$.

In this framework an interesting issue is the effect of a single crack on the structural response [3-6]. For the dynamic analysis the presence of the crack is usually neglected in the evaluation of the mass and damping, so only the stiffness is affected by the crack. This study is performed adopting a finite element model for the damaged beam with an on-edge nonpropagating crack. Undamaged elements of the beam are modeled by Euler-type finite elements.

It is impossible to be certain of the crack depth, consequently this quantity should be assumed uncertain. Therefore, it is important to estimate the effect of this uncertainty on the structural dynamic response [7]. However, while the numerous available data permit to model with good accuracy the excitations as stochastic processes, unfortunately the data about the structural parameters are quite limited. It follows that the probabilistic approach cannot be realistically applied to represent structural uncertainties; indeed, it requires a wealth of data, often unavailable, to define the probability distribution density of the fluctuating structural parameters. For this reason the uncertain crack depth is here modeled as an interval variable [8]: in this model the uncertainty is defined by the knowledge of the lower and upper bound only.

The aim of this paper is to determine the lower and upper bounds of the response of damaged beams with uncertain-but-bounded crack subjected to both deterministic and stochastic excitations modeled as zero mean Gaussian random processes. To this aim the method proposed by Muscolino and Sofi [9] is extended to cracked beams. Specifically, the method adopts an improvement of the classical interval analysis [8], introducing a particular unitary interval, called Extra Unitary Interval (EUI) to split both the time dependent deterministic and random response, in modal subspace, as sum of two aliquots: the midpoint or nominal solution and the deviation. For stochastic excitations the Kronecker algebra [10] is adopted to derive the differential equations governing the time-evolution of the midpoint and deviation covariance vectors of the response under zero-mean Gaussian stochastic input process.

Once such equations are solved, the upper and lower bounds of the deterministic response as well as the covariance vector are evaluated by applying handy formulas.

In particular the proposed procedure is based on the following main steps: i) to define a finite element model of the beam in which the model of fully open crack is used to represent the damaged element; ii) to model the crack depth as an interval variable; iii) to evaluate in time domain the response for deterministic and stochastic excitation, by adopting an unified approach based on the Improved Interval Analysis via EUI [9].

Numerical results concerning a cracked beam with uncertain-but-bounded stiffness properties under deterministic and uniformly modulated white noise excitation are presented to show the effectiveness of the proposed method.

\section{CRACK MODEL DESCRIPTION}

The adopted mathematical model used for the damaged beam with a transverse on-edge non-propagating crack is based on the finite element model proposed in Refs. [1,2].

According to Saint-Venant principle only the element that contains a central crack is modified, being the stress field affected only in the region adjacent to the crack. Such a perturba- 
tion of the stress field is relevant especially when the crack is open and determines a local reduction of the flexural rigidity.

It follows that the element stiffness matrix, with the exception of the terms which represent the cracked element, may be regarded as unchanged under a certain limitation of the element size.

Undamaged parts of the beam are modelled by Euler type finite elements with two nodes and two degrees of freedom (transverse displacement and rotation) at each node.

Neglecting shear action, the strain energy of an element without a crack can be written as

$$
W^{(0)}=\frac{1}{2 E I} \int_{0}^{\ell}(M+P z)^{2} d z=\frac{1}{2 E I}\left(M^{2} \ell+\frac{P^{2} \ell^{3}}{3}+M P \ell^{2}\right)
$$

where $E$ is the Young's modulus, $\ell$ the length of the finite element, $P$ and $M$ are the shear and bending internal forces at the right node of the element and $I$ is the moment of inertia.

Fracture mechanics studied the calculation of the additional stress energy of a crack providing flexibility coefficients expressed by a stress intensity factor in the linear elastic range, using Castigliano's theorem.

Concerning a rectangular beam having width $b$ and thickness $h$ the additional energy due to the crack can be expressed as

$$
W^{(1)}=b \int_{0}^{a}\left(\frac{K_{I}^{2}+K_{I I}^{2}}{E^{\prime}}+\frac{(1+v) K_{I I I}^{2}}{E}\right) d a
$$

where $a$ is the crack depth, $E^{\prime}=E$ for plane stress and $E^{\prime}=E /\left(1-v^{2}\right)$ for plane strain, $v$ is the Poisson ratio and $K_{I}, K_{I I}, K_{I I I}$ are stress intensity factors for opening type, sliding type and tearing cracks, respectively.

Taking into account only bending, Eq.(2) leads to

$$
W^{(1)}=b \int_{0}^{a} \frac{\left(K_{I M}+K_{I P}\right)^{2}+K_{I I P}^{2}}{E^{1}} d a
$$

where

$$
K_{I M}=\frac{6 M}{b h^{2}} \sqrt{\pi a} F_{I}(s) ; \quad K_{I P}=\frac{3 P \ell}{b h^{2}} \sqrt{\pi a} F_{I}(s) ; \quad K_{I I P}=\frac{P}{b h} \sqrt{\pi a} F_{I I}(s)
$$

are stress intensity factors for opening-type and sliding-type cracks due to $M$ and $P$, respectively, and

$$
\begin{aligned}
& F_{I}(s)=\sqrt{\frac{2}{\pi s} \tan \left(\frac{\pi s}{2}\right)} \frac{0.923+0.199[1-\sin (\pi s / 2)]^{4}}{\cos (\pi s / 2)} \\
& F_{I I}(s)=\left(3 s-2 s^{2}\right) \frac{1.122-0.561 \mathrm{~s}+0.085 s^{2}+0.18 s^{3}}{\sqrt{1-s}}
\end{aligned}
$$

with $s=a / h$ is the ratio between the crack depth and the height of the element.

The generic component $d_{i j}^{(0)}$ of the compliance (or flexibility) matrix $\mathbf{D}_{\mathrm{e}}^{(0)}$ of the undamaged element can be derived as 


$$
d_{i j}^{(0)}=\frac{\partial^{2} W^{(0)}}{\partial P_{i} \partial P_{j}}, \quad i, j=1,2 ; \quad P_{1}=P, \quad P_{2}=M
$$

whereas the terms $d_{i j}^{(1)}$ of the additional flexibility matrix $\mathbf{D}_{\mathrm{e}}^{(1)}$ due to the crack can be formulated by:

$$
d_{i j}^{(1)}=\frac{\partial^{2} W^{(1)}}{\partial P_{i} \partial P_{j}}, \quad i, j=1,2 ; \quad P_{1}=P, \quad P_{2}=M
$$

Finally the total flexibility matrix for the element with an open crack is:

$$
\mathbf{D}_{\mathrm{e}}=\mathbf{D}_{\mathrm{e}}^{(0)}+\mathbf{D}_{\mathrm{e}}^{(1)}
$$

From the equilibrium condition it follows

$$
\left(P_{i} M_{i} P_{i+1} M_{i+1}\right)^{T}=\mathbf{T}\left(P_{i+1} M_{i+1}\right)^{T}
$$

where the apex $T$ means transpose matrix and

$$
\mathbf{T}^{T}=\left[\begin{array}{cccc}
-1 & -\ell & 1 & 0 \\
0 & -1 & 0 & 1
\end{array}\right]
$$

By the principle of virtual work the stiffness matrix of the undamaged element takes the following form:

$$
\mathbf{K}_{\mathrm{e}}=\mathbf{T D}_{\mathrm{e}}^{(0)-1} \mathbf{T}^{T}
$$

while the stiffness matrix of the cracked element may be derived a

$$
\mathbf{K}_{\mathrm{c}, \mathrm{e}}=\mathbf{T D}_{\mathrm{e}}^{-1} \mathbf{T}^{T}
$$

Once the stiffness matrices of the undamaged and cracked elements are defined, for the beam discretized in $N_{e}$ finite elements the stiffness matrix $\mathbf{K}$ of order $n \times n$ with $n=2 N_{\mathrm{e}}$ can be straightforwardly evaluated following the classical assembly rules.

Moreover, in the framework of the finite element approximation, it is usually assumed that the crack does not modify the mass distribution.

\section{GOVERNING EQUATIONS FOR UNCERTAIN-BUT-BOUNDED CRACK DEPTH}

The equation of motion of a quiescent damaged beam discretized by $N_{e}$ finite elements subjected to an external excitation $\mathbf{f}(t)$ with uncertain crack depth modeled as interval variable can be written as:

$$
\mathbf{M} \ddot{\mathbf{u}}(\alpha, t)+\mathbf{C}\left(a_{0}, \alpha\right) \dot{\mathbf{u}}(\alpha, t)+\mathbf{K}\left(a_{0}, \alpha\right) \mathbf{u}(\alpha, t)=\mathbf{f}(t), \quad \alpha \in \alpha^{I}=[\underline{\alpha}, \bar{\alpha}]
$$

where $\mathbf{M}$ is the $n \times n$ mass matrix of the structure, $\mathbf{C}\left(a_{0}, \alpha\right)$ is the $n \times n$ damping matrix, $\mathbf{K}\left(a_{0}, \alpha\right)$ is the $n \times n$ stiffness matrix and $\mathbf{f}(t)$ is the vector function of order $n \times 1 ; \mathbf{u}(\alpha, t)$ is the interval vector of nodal displacements of order $n \times 1$ and a dot over a variable denotes differentiation with respect to time $t$.

The $n \times n$ interval stiffness matrix $\mathbf{K}\left(a_{0}, \alpha\right)$ is here expressed as a function of the uncertain structural parameter $\alpha$ as follows: 


$$
\mathbf{K}\left(a_{0}, \alpha\right)=\mathbf{K}_{\mathrm{C}}\left(a_{0}\right)+\alpha \mathbf{K}_{1}\left(a_{0}\right) ; \quad \mathbf{K}_{1}\left(a_{0}\right)=\left.\frac{\partial \mathbf{K}\left(a_{0}, \alpha\right)}{\partial \alpha}\right|_{\alpha=0}
$$

where $\alpha$ is the dimensionless fluctuation of the uncertain crack depth $a=a_{0}(1+\alpha)$ with $a_{0}$ its mean value. In Eq.(14) $\mathbf{K}_{\mathrm{C}}\left(a_{0}\right)$ is the midpoint stiffness matrix. It is a positive definite symmetric matrix of order $n \times n$, while $\mathbf{K}_{1}\left(a_{0}\right)$ is a symmetric matrix of order $n \times n$ and rank $r$.The Rayleigh model is herein adopted for the interval damping matrix, i.e.:

$$
\mathbf{C}\left(a_{0}, \alpha\right)=c_{0} \mathbf{M}+c_{1} \mathbf{K}\left(a_{0}, \alpha\right)=c_{0} \mathbf{M}+c_{1} \mathbf{K}_{\mathrm{C}}\left(a_{0}\right)+c_{1} \alpha \mathbf{K}_{1}\left(a_{0}\right)=\mathbf{C}_{\mathrm{C}}\left(a_{0}\right)+\alpha \mathbf{C}_{1}\left(a_{0}\right)
$$

where $c_{0}$ and $c_{1}$ are the Rayleigh damping constants having units $s^{-1}$ and $s$, respectively. Hereafter we indicate $\mathbf{K}_{\mathrm{C}}\left(a_{0}\right)=\mathbf{K}_{\mathrm{C}}, \mathbf{K}_{1}\left(a_{0}\right)=\mathbf{K}_{1}$ and $\mathbf{C}_{\mathrm{C}}\left(a_{0}\right)=\mathbf{C}_{\mathrm{C}}$ for sake of notation compactness.

According to the Interval Analysis [8], the interval real number $\alpha^{I} \triangleq[\underline{\alpha}, \bar{\alpha}] \in \mathbb{R} \mathbb{R}$ such that $\underline{\alpha} \leq \alpha \leq \bar{\alpha}$, with $\underline{\alpha}$ and $\bar{\alpha}$ denoting the lower and upper bound of $\alpha$, is introduced. In this case the midpoint (or mean) and deviation amplitude (or radius) can be written as:

$$
\alpha_{0}=\frac{\bar{\alpha}+\underline{\alpha}}{2} ; \Delta \alpha=\frac{\bar{\alpha}-\underline{\alpha}}{2}>0
$$

Introducing the so-called extra symmetric unitary interval (EUI) [9] variable $\hat{e}_{\alpha}^{I} \triangleq[-1,1]$ the following affine form definition of the interval variable $\alpha$ is provided:

$$
\alpha=\alpha_{0}+\Delta \alpha \hat{e}_{\alpha}^{I}
$$

Moreover the dimensionless fluctuation $\alpha$ of the uncertain-but-bounded crack depth $a=a_{0}(1+\alpha)$ around its mean value $a_{0}$ can be modeled as symmetric interval in such a way $\alpha \in[\underline{\alpha}, \bar{\alpha}]$ with $\bar{\alpha}=-\underline{\alpha}$. Under this assumption Eq.(17) reduces to $\alpha=\Delta \alpha \hat{e}_{\alpha}^{I}$ and the $n \times n$ interval stiffness matrix $\mathbf{K}^{I}\left(\mathrm{a}_{0}, \alpha\right)$ can be rewritten in the form:

$$
\mathbf{K}^{I}\left(a_{0}, \alpha\right)=\mathbf{K}_{\mathrm{C}}+\Delta \alpha \hat{e}_{\alpha}^{I} \mathbf{K}_{1}
$$

where the matrices $\mathbf{K}_{\mathrm{C}}$ and $\mathbf{K}_{1}$ have been previously defined in Eq.(14).

In the framework of the traditional modal analysis, the solution of the equations of motion (13) may be pursued by introducing the following coordinate transformation:

$$
\mathbf{u}(\alpha, t)=\boldsymbol{\Phi}_{\mathrm{C}} \mathbf{q}(\alpha, t), \quad \alpha \in \alpha^{I}=[\underline{\alpha}, \bar{\alpha}]
$$

where $\mathbf{q}(\alpha, t)$ is the interval vector gathering the first $m$ modal coordinates $q_{j}(\alpha, t)$ $(j=1,2, \ldots, m) ; \boldsymbol{\Phi}_{\mathrm{C}}$ is the modal matrix, of order $n \times m$, pertaining to the midpoint configuration. Specifically, the modal matrix $\boldsymbol{\Phi}_{\mathrm{C}}$, collecting the first $m$ eigenvectors normalized with respect to the mass matrix $\mathbf{M}$, is evaluated as solution of the following eigenproblem:

$$
\mathbf{K}_{\mathrm{C}} \boldsymbol{\Phi}_{\mathrm{C}}=\mathbf{M} \boldsymbol{\Phi}_{\mathrm{C}} \boldsymbol{\Omega}_{\mathrm{C}}^{2} ; \quad \boldsymbol{\Phi}_{\mathrm{C}}^{T} \mathbf{M} \boldsymbol{\Phi}_{\mathrm{C}}=\mathbf{I}_{m} ; \quad \boldsymbol{\Phi}_{\mathrm{C}}^{T} \mathbf{K}_{\mathrm{C}} \boldsymbol{\Phi}_{\mathrm{C}}=\boldsymbol{\Omega}_{\mathrm{C}}^{2}
$$


$\mathbf{I}_{m}$ being the identity matrix of order $m$ and $\mathbf{\Omega}_{\mathrm{C}}^{2}$ the spectral matrix listing the squares of the natural circular frequencies of the structure related to the mean value of the uncertain crack depth and the apex $T$ means transpose matrix.

By applying the coordinate transformation (19), the equations of motion can be projected in the modal space:

$$
\ddot{\mathbf{q}}(\alpha, t)+\mathbf{\Xi}(\alpha) \dot{\mathbf{q}}(\alpha, t)+\mathbf{\Omega}^{2}(\alpha) \mathbf{q}(\alpha, t)=\boldsymbol{\Phi}_{\mathrm{C}}^{T} \mathbf{f}(t), \quad \alpha \in \alpha^{I}=[\underline{\alpha}, \bar{\alpha}]
$$

where $\boldsymbol{\Omega}^{2}(\alpha)=\boldsymbol{\Phi}_{\mathrm{C}}^{T} \mathbf{K}(\alpha) \boldsymbol{\Phi}_{\mathrm{C}}$ and $\boldsymbol{\Xi}(\alpha)=\boldsymbol{\Phi}_{\mathrm{C}}^{T} \mathbf{C}(\alpha) \boldsymbol{\Phi}_{\mathrm{C}}$ is the generalized damping matrix. Based on Eq. (18), the matrix $\mathbf{\Omega}^{2}(\alpha)$ can be split as

$$
\begin{aligned}
& \boldsymbol{\Omega}^{2}(\alpha)=\boldsymbol{\Phi}_{\mathrm{C}}^{T} \mathbf{K}(\alpha) \boldsymbol{\Phi}_{\mathrm{C}}=\boldsymbol{\Omega}_{\mathrm{C}}^{2}+\Delta \alpha \hat{e}_{\alpha}^{I} \boldsymbol{\Omega}_{1}^{2}, \alpha \in \alpha^{I}=[\underline{\alpha}, \bar{\alpha}] ; \\
& \boldsymbol{\Omega}_{\mathrm{C}}^{2}=\boldsymbol{\Phi}_{\mathrm{C}}^{T} \mathbf{K}_{\mathrm{C}} \boldsymbol{\Phi}_{\mathrm{C}} ; \boldsymbol{\Omega}_{1}^{2}=\boldsymbol{\Phi}_{\mathrm{C}}^{T} \mathbf{K}_{1} \boldsymbol{\Phi}_{\mathrm{C}} .
\end{aligned}
$$

where the matrix $\boldsymbol{\Omega}_{1}^{2}$ is not a diagonal matrix.

Analogously the matrix $\Xi(\alpha)$ can be split as

$$
\begin{aligned}
& \boldsymbol{\Xi}(\alpha)=\boldsymbol{\Phi}_{\mathrm{C}}^{T} \mathbf{C}(\alpha) \boldsymbol{\Phi}_{\mathrm{C}}=\boldsymbol{\Xi}_{\mathrm{C}}+\Delta \alpha \hat{e}_{\alpha}^{I} \boldsymbol{\Xi}_{1}, \alpha \in \alpha^{I}=[\underline{\alpha}, \bar{\alpha}] ; \\
& \boldsymbol{\Xi}_{\mathrm{C}}=c_{0} \mathbf{I}_{m}+c_{1} \mathbf{\Omega}_{\mathrm{C}}^{2} ; \quad \boldsymbol{\Xi}_{1}=c_{1} \boldsymbol{\Phi}_{\mathrm{C}}^{T} \mathbf{K}_{1} \boldsymbol{\Phi}_{\mathrm{C}} .
\end{aligned}
$$

where only the matrix $\boldsymbol{\Xi}_{\mathrm{C}}$ is a diagonal one.

\section{TIME DOMAIN RESPONSE}

\subsection{Deterministic excitation}

The key idea is to evaluate two response vectors [9] associated respectively to LB of $\hat{e}_{\alpha}^{I}$ and to UB of $\hat{e}_{\alpha}^{I}$.

The first one, denoted by $\mathbf{q}^{-}(\alpha, t)$, is evaluated by solving the following differential set of equations:

$$
\ddot{\mathbf{q}}^{-}(\alpha, t)+\left[\boldsymbol{\Xi}_{\mathrm{C}}-\Delta \alpha \boldsymbol{\Xi}_{1}\right] \dot{\mathbf{q}}^{-}(\alpha, t)+\left[\mathbf{\Omega}_{\mathrm{C}}^{2}-\Delta \alpha \mathbf{\Omega}_{1}^{2}\right] \mathbf{q}^{-}(\alpha, t)=\boldsymbol{\Phi}_{\mathrm{C}}^{T} \mathbf{f}(t)
$$

while the second, denoted by $\mathbf{q}^{+}(\alpha, t)$, is evaluated by solving the following differential set of equations:

$$
\ddot{\mathbf{q}}^{+}(\alpha, t)+\left[\boldsymbol{\Xi}_{\mathrm{C}}+\Delta \alpha \boldsymbol{\Xi}_{1}\right] \dot{\mathbf{q}}^{+}(\alpha, t)+\left[\boldsymbol{\Omega}_{\mathrm{C}}^{2}+\Delta \alpha \mathbf{\Omega}_{1}^{2}\right] \mathbf{q}^{+}(\alpha, t)=\boldsymbol{\Phi}_{\mathrm{C}}^{T} \mathbf{f}(t)
$$

Notice that the two set of Eqs. (24) and (25) cannot decoupled by a real coordinate of transformation, then they are referred in literature as non-classically damped systems. It has been recognized that in order to evaluate the response of non-classically damped systems the state variables have to be introduced [11].

In state variables the previous equations can be rewritten as:

$$
\dot{\mathbf{y}}^{\mp}(\alpha, t)=\mathbf{D}^{\mp}(\alpha) \mathbf{y}^{\mp}(\alpha, t)+\mathbf{V} \mathbf{f}(t)
$$

being 


$$
\begin{gathered}
\mathbf{y}^{\mp}(\alpha, t)=\left[\begin{array}{c}
\mathbf{q}^{\mp}(\alpha, t) \\
\dot{\mathbf{q}}^{\mp}(\alpha, t)
\end{array}\right] ; \mathbf{D}^{-}(\alpha)=\left[\begin{array}{cc}
\mathbf{0} & \mathbf{I}_{m} \\
-\left[\mathbf{\Omega}_{\mathrm{C}}^{2}-\Delta \alpha \mathbf{\Omega}_{1}^{2}\right] & -\left[\boldsymbol{\Xi}_{\mathrm{C}}-\Delta \alpha \mathbf{\Xi}_{1}\right]
\end{array}\right] ; \\
\mathbf{D}^{+}(\alpha)=\left[\begin{array}{cc}
\mathbf{0} & \mathbf{I}_{m} \\
-\left[\mathbf{\Omega}_{\mathrm{C}}^{2}+\Delta \alpha \mathbf{\Omega}_{1}^{2}\right] & -\left[\boldsymbol{\Xi}_{\mathrm{C}}+\Delta \alpha \mathbf{\Xi}_{1}\right]
\end{array}\right] ; \mathbf{V}=\left[\begin{array}{c}
\mathbf{0} \\
\mathbf{\Phi}_{\mathrm{C}}^{T}
\end{array}\right]
\end{gathered}
$$

where $\mathbf{0}$ is the zero matrix.

The solution of these equations can be obtained by applying the numerical procedure proposed by [11] writing:

$$
\mathbf{y}^{\mp}\left(\alpha, t_{k+1}\right)=\boldsymbol{\Theta}^{\mp}(\alpha, \Delta t) \mathbf{y}^{\mp}\left(\alpha, t_{k}\right)+\gamma_{0}^{\mp}(\alpha, \Delta t) \mathbf{V} \mathbf{f}\left(t_{k}\right)+\gamma_{1}^{\mp}(\alpha, \Delta t) \mathbf{V} \mathbf{f}\left(t_{k+1}\right)
$$

where

$$
\begin{aligned}
& \boldsymbol{\Theta}^{\mp}(\alpha, \Delta t)=\exp \left[\Delta t \mathbf{D}^{\mp}(\alpha)\right] \\
& \mathbf{L}^{\mp}(\Delta t)=\left[\boldsymbol{\Theta}^{\mp}(\alpha, \Delta t)-\mathbf{I}_{2 m}\right]\left(\mathbf{D}^{\mp}(\alpha)\right)^{-1} ; \\
& \boldsymbol{\gamma}_{0}^{\mp}(\alpha, \Delta t)=\left[\boldsymbol{\Theta}^{\mp}(\alpha, \Delta t)-\frac{1}{\Delta t} \mathbf{L}^{\mp}(\alpha, \Delta t)\right]\left(\mathbf{D}^{\mp}(\alpha)\right)^{-1} ; \\
& \boldsymbol{\gamma}_{1}^{\mp}(\alpha, \Delta t)=\left[\frac{1}{\Delta t} \mathbf{L}^{\mp}(\alpha, \Delta t)-\mathbf{I}_{2 m}\right]\left(\mathbf{D}^{\mp}(\alpha)\right)^{-1}
\end{aligned}
$$

Once the vectors $\mathbf{q}^{-}(\alpha, t)$ and $\mathbf{q}^{+}(\alpha, t)$ have been evaluated, the corresponding nodal responses can be written as:

$$
\mathbf{u}^{-}(\alpha, t)=\boldsymbol{\Phi}_{\mathrm{C}} \mathbf{q}^{-}(\alpha, t) ; \quad \mathbf{u}^{+}(\alpha, t)=\boldsymbol{\Phi}_{\mathrm{C}} \mathbf{q}^{+}(\alpha, t)
$$

In order to evaluate the lower bound (LB), $\underline{\mathbf{u}}(\alpha, t)$, and upper bound (UB), $\overline{\mathbf{u}}(\alpha, t)$, of the interval dynamic response, $\mathbf{u}^{I}(\alpha, t)$, let denote with $u_{k}^{I}(t)$ the $k$-th element of the vector $\mathbf{u}^{I}(\alpha, t)$, and with $u_{k}^{-}(t)$ and $u_{k}^{+}(t)$ the $k$-th elements of the vectors $\mathbf{u}^{-}(\alpha, t)$ and $\mathbf{u}^{+}(\alpha, t)$, respectively. Then the lower and upper bounds of the $k$-th nodal dynamic response in the time domain can be written as:

$$
\underline{u}_{k}(t)=\min \left\{u_{k}^{-}(t), u_{k}^{+}(t)\right\} ; \quad \bar{u}_{k}(t)=\max \left\{u_{k}^{-}(t), u_{k}^{+}(t)\right\}
$$

where $\min \{\bullet\}$ and $\max \{\bullet\}$ means minimum and maximum values of the quantity into curly parentheses, respectively.

Once the LB and UB functions are evaluated, the midpoint function can be evaluated as:

$$
u_{k, \text { mid }}(t)=\frac{\underline{u}_{k}(t)+\bar{u}_{k}(t)}{2}
$$

\subsection{Stochastic excitation}

The statistics of the response of a damaged beam with uncertain-but-bounded crack depth under random excitation turn out to be represented by time-dependent stochastic interval vectors. In the following, a method for determining the upper and lower bounds of second-order statistics will be developed. 
Without loss of generality, the forcing term in Eq.(13) is herein assumed to be a monocorrelated zero-mean Gaussian random vector process, appropriately defined as follows:

$$
\mathbf{f}(t)=F(t) \mathbf{s}
$$

where $\mathbf{s}$ is a $n$-order vector listing the spatial distribution of loads and $F(t)$ is a zero-mean Gaussian non-stationary uniformly modulated random process, fully characterized by the autocorrelation function $R_{F F}\left(t_{1}, t_{2}\right)$.

As known, under the assumptions of linear elastic behaviour and uncertain-but-bounded structural parameter, namely the crack depth, the response process of a structural system excited by a zero-mean Gaussian random process is zero-mean Gaussian too. The covariance matrix is defined as:

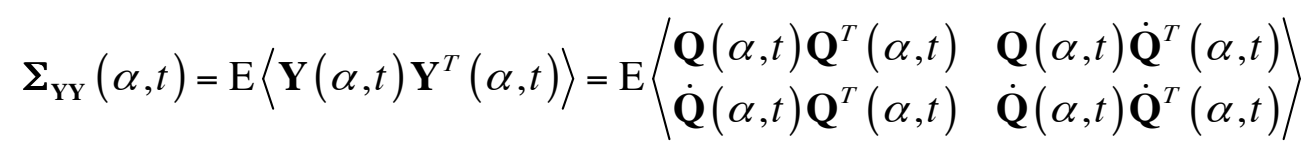

$\mathrm{E}\langle\bullet\rangle$ being the mathematical expectation operator.

Alternatively, the stochastic response can defined in a more suitable form by introducing the covariance vector $\sigma_{\mathbf{Y}}(\alpha, t)$ :

$$
\boldsymbol{\sigma}_{\mathbf{Y}}(\alpha, t)=\mathrm{E}\langle\mathbf{Y}(\alpha, t) \odot \mathbf{Y}(\alpha, t)\rangle=\operatorname{Vec}\left\{\mathrm{E}\left\langle\mathbf{Y}(\alpha, t) \mathbf{Y}^{T}(\alpha, t)\right\rangle^{T}\right\}=\operatorname{Vec}\left\{\boldsymbol{\Sigma}_{\mathbf{Y Y}}^{T}(\alpha, t)\right\}
$$

where the symbol $\odot$ denotes the block Kronecker product [10]. The vector $\boldsymbol{\sigma}_{\mathbf{Y}}(\alpha, t)$ represents the block vectorialized form ("Vec $\{\cdot\}$ ") of the covariance matrix, that is a column vector defined in such a way that the columns of the sub-matrices forming the matrix in parentheses are written one below each other.

After some algebra, the differential equations governing the time-evolution of the vector $\boldsymbol{\sigma}_{\mathbf{Y}}(\alpha, t)$ can be written as:

$$
\dot{\boldsymbol{\sigma}}_{\mathbf{Y}}^{\mp}(\alpha, t)=\mathbf{D}_{2}^{\mp}(\alpha) \boldsymbol{\sigma}_{\mathbf{Y}}^{\mp}(\alpha, t)+\mathbf{F}_{\mathbf{Y}}^{\mp}(\alpha, t)
$$

where

$$
\begin{aligned}
& \boldsymbol{\sigma}_{\mathbf{Y}}^{\mp}(\alpha, t)=\left[\begin{array}{c}
\boldsymbol{\sigma}_{\mathbf{Q Q}}^{\mp}(\alpha, t) \\
\boldsymbol{\sigma}_{\mathbf{Q Q}}^{\mp}(\alpha, t) \\
\boldsymbol{\sigma}_{\mathbf{Q Q}}^{\mp}(\alpha, t) \\
\boldsymbol{\sigma}_{\mathbf{Q Q}}^{\mp}(\alpha, t)
\end{array}\right] ; \\
& \mathbf{D}_{2}^{\mp}(\alpha)=\mathbf{D}^{\mp}(\alpha) \odot \mathbf{I}_{2 m}+\mathbf{I}_{2 m} \odot \mathbf{D}^{\mp}(\alpha) ; \\
& \mathbf{F}_{\mathbf{Y}}^{\mp}(\alpha, t)=\mathbf{V}_{2} \mathrm{E}\left\langle\mathbf{Y}^{\mp}(\alpha, t) F(t)\right\rangle
\end{aligned}
$$

with

$$
\mathbf{V}_{2}=\left[\left(\mathbf{V s} \odot \mathbf{I}_{2 m}\right)+\left(\mathbf{I}_{2 m} \odot \mathbf{V s}\right)\right]
$$


The matrices $\mathbf{D}^{-}(\alpha)$ and $\mathbf{D}^{+}(\alpha)$ required to define $\mathbf{D}_{2}^{\mp}(\alpha)$ have been previously defined in Eq.(27). Taking into account the formal analogy between Eq.(36) and the equation of motion in the modal state variable space (26), the response can be evaluate numerically as:

$$
\left.\boldsymbol{\sigma}_{\mathbf{Y}}^{\mp}\left(\alpha, t_{k+1}\right)=\boldsymbol{\Theta}_{2}^{\mp}(\alpha, \Delta t) \boldsymbol{\sigma}_{\mathbf{Y}}^{\mp}\left(\alpha, t_{k}\right)\right)+\boldsymbol{\gamma}_{0,2}^{\mp}(\alpha, \Delta t) \mathbf{F}_{\mathbf{Y}}^{\mp}\left(\alpha, t_{k}\right)+\boldsymbol{\gamma}_{1,2}^{\mp}(\alpha, \Delta t) \mathbf{F}_{\mathbf{Y}}^{\mp}\left(\alpha, t_{k+1}\right)
$$

where

$$
\begin{aligned}
& \boldsymbol{\Theta}_{2}^{\mp}(\alpha, \Delta t)=\boldsymbol{\Theta}^{\mp}(\alpha, \Delta t) \odot \boldsymbol{\Theta}^{\mp}(\alpha, \Delta t) ; \\
& \mathbf{L}_{2}^{\mp}(\alpha, \Delta t)=\left[\boldsymbol{\Theta}_{2}^{\mp}(\alpha, \Delta t)-\mathbf{I}_{(2 m)^{2}}\right]\left(\mathbf{D}_{2}^{\mp}(\alpha)\right)^{-1} ; \\
& \boldsymbol{\gamma}_{0,2}^{\mp}(\alpha, \Delta t)=\left[\boldsymbol{\Theta}_{2}^{\mp}(\alpha, \Delta t)-\frac{1}{\Delta t} \mathbf{L}_{2}^{\mp}(\alpha, \Delta t)\right]\left(\mathbf{D}_{2}^{\mp}(\alpha)\right)^{-1} ; \\
& \boldsymbol{\gamma}_{1,2}^{\mp}(\alpha, \Delta t)=\left[\frac{1}{\Delta t} \mathbf{L}_{2}^{\mp}(\alpha, \Delta t)-\mathbf{I}_{(2 m)^{2}}\right]\left(\mathbf{D}_{2}^{\mp}(\alpha)\right)^{-1} ; \\
& \mathbf{F}_{\mathbf{Y}}^{\mp}(\alpha, t)=\mathbf{V}_{2} \mathrm{E}\left\langle\mathbf{Y}^{\mp}(\alpha, t) F(t)\right\rangle=\mathbf{V}_{2} \int_{0}^{t} \boldsymbol{\Theta}^{\mp}(\alpha, t-\tau) \mathbf{V} \mathbf{s} R_{F F}(t, \tau) \mathrm{d} \tau
\end{aligned}
$$

A procedure proposed to evaluate the inverse of the matrices $\mathbf{D}_{2}^{\mp}(\alpha)$ in Eq.(40), name$\operatorname{ly}\left(\mathbf{D}_{2}^{\mp}(\alpha)\right)^{-1}$, moves from the evaluation of $\psi_{j}^{\mp}$ and $\lambda_{j}^{\mp}$ being the $j$-th eigenvector and the associated eigenvalue, solutions of the following eigenproblems:

$$
\mathbf{D}^{\mp} \boldsymbol{\Psi}^{\mp}=\boldsymbol{\Psi}^{\mp} \boldsymbol{\Lambda}^{\mp} ; \quad \boldsymbol{\Psi}^{\mp T} \mathbf{A}^{\mp} \boldsymbol{\Psi}^{\mp}=\mathbf{I}_{r}
$$

solved respectively for the matrices $\mathbf{D}^{-}(\alpha)$ and $\mathbf{D}^{+}(\alpha)$ with

$$
\boldsymbol{\Psi}^{\mp}=\left[\begin{array}{llll}
\boldsymbol{\Psi}_{1}^{\mp} & \boldsymbol{\psi}_{2}^{\mp} & \cdots & \boldsymbol{\Psi}_{r}^{\mp}
\end{array}\right] ; \boldsymbol{\Lambda}^{\mp}=\operatorname{Diag}\left[\lambda_{1}^{\mp}, \quad \lambda_{2}^{\mp}, \quad \ldots \quad \lambda_{r}^{\mp}\right]
$$

and

$$
\mathbf{A}^{\mp}(\alpha)=\left[\begin{array}{cc}
{\left[\boldsymbol{\Xi}_{\mathrm{C}} \mp \Delta \alpha \boldsymbol{\Xi}_{1}\right]} & \mathbf{I}_{m} \\
\mathbf{I}_{m} & \mathbf{0}
\end{array}\right]
$$

The evaluation of the complex diagonal matrices $\boldsymbol{\Lambda}_{2}^{\mp}$ defined as

$$
\boldsymbol{\Lambda}_{2}^{\mp}=\boldsymbol{\Lambda}^{\mp} \odot \mathbf{I}_{2 \mathrm{~m}}+\mathbf{I}_{2 \mathrm{~m}} \odot \boldsymbol{\Lambda}^{\mp}
$$

allows to calculate the matrices $\left(\mathbf{D}_{2}^{\mp}(\alpha)\right)^{-1}$ as follows:

$$
\left(\mathbf{D}_{2}^{\mp}(\alpha)\right)^{-1}=\left(\boldsymbol{\Psi}^{\mp} \odot \boldsymbol{\Psi}^{\mp}\right)\left(\boldsymbol{\Lambda}_{2}^{\mp}\right)^{-1}\left(\boldsymbol{\Psi}^{\mp T} \mathbf{A}^{\mp}(\alpha) \odot \boldsymbol{\Psi}^{\mp T} \mathbf{A}^{\mp}(\alpha)\right)
$$

Once the modal covariances vectors $\boldsymbol{\sigma}_{\mathbf{Y}}^{-}(\alpha, t)$ and $\boldsymbol{\sigma}_{\mathbf{Y}}^{+}(\alpha, t)$ have been evaluated, the nodal corresponding quantities can be evaluated as follows: 


$$
\boldsymbol{\sigma}_{\mathbf{Z}}^{-}(\alpha, t)=\left[\begin{array}{c}
\boldsymbol{\sigma}_{\mathbf{U}}^{-}(\alpha, t) \\
\boldsymbol{\sigma}_{\dot{\mathbf{U}} \mathbf{U}}^{-}(\alpha, t) \\
\boldsymbol{\sigma}_{\mathbf{U} \mathbf{U}}^{-}(\alpha, t) \\
\boldsymbol{\sigma}_{\dot{\mathbf{U}} \mathbf{U}}^{-}(\alpha, t)
\end{array}\right]=\boldsymbol{\Pi}_{\mathrm{C}} \boldsymbol{\sigma}_{\mathbf{Y}}^{-}(\alpha, t) ; \boldsymbol{\sigma}_{\mathbf{Z}}^{+}(\alpha, t)=\left[\begin{array}{c}
\boldsymbol{\sigma}_{\mathbf{U} \mathbf{U}}^{+}(\alpha, t) \\
\boldsymbol{\sigma}_{\dot{\mathbf{U}}}^{+}(\alpha, t) \\
\boldsymbol{\sigma}_{\mathbf{U}}^{+}(\alpha, t) \\
\boldsymbol{\sigma}_{\dot{\mathbf{U}} \mathbf{U}}^{+}(\alpha, t)
\end{array}\right]=\boldsymbol{\Pi}_{\mathrm{C}} \boldsymbol{\sigma}_{\mathbf{Y}}^{+}(\alpha, t)
$$

where

$$
\boldsymbol{\Pi}_{\mathrm{C}}=\left[\begin{array}{cc}
\boldsymbol{\Phi}_{\mathrm{C}} & \mathbf{0} \\
\mathbf{0} & \boldsymbol{\Phi}_{\mathrm{C}}
\end{array}\right] \odot\left[\begin{array}{cc}
\boldsymbol{\Phi}_{\mathrm{C}} & \mathbf{0} \\
\mathbf{0} & \boldsymbol{\Phi}_{\mathrm{C}}
\end{array}\right]
$$

The lower bound (LB) and upper bound (UB) of the $k$-th nodal interval variance of the dynamic response can be evaluated as a function of the $(k-1) m+k$-th element of vectors $\boldsymbol{\sigma}_{\mathbf{U}}^{-}(\alpha, t)$ and $\boldsymbol{\sigma}_{\mathbf{U}}^{+}(\alpha, t)$, that is:

$$
\begin{aligned}
& \underline{\sigma}_{U_{k}}^{2}(t)=\min \left\{\boldsymbol{\sigma}_{\mathbf{U}}^{-}(\alpha, t), \boldsymbol{\sigma}_{\mathbf{U}}^{+}(\alpha, t)\right\}_{(k-1) n+k} \\
& \bar{\sigma}_{U_{k}}^{2}(t)=\max \left\{\boldsymbol{\sigma}_{\mathbf{U}}^{-}(\alpha, t), \boldsymbol{\sigma}_{\mathbf{U}}^{+}(\alpha, t)\right\}_{(k-1) n+k}
\end{aligned}
$$

where $\min \{\bullet\}_{(k-1) m+k}$ and $\max \{\bullet\}_{(k-1) m+k}$ means minimum and maximum values of the $(k-1) m+k$-th element of the vectors into curly parentheses, respectively. Once the LB and UB functions are evaluated the midpoint vector can be evaluated as:

$$
\sigma_{U_{k}, \text { mid }}^{2}(\alpha, t)=\frac{\underline{\sigma}_{U_{k}}^{2}(\alpha, t)+\bar{\sigma}_{U_{k}}^{2}(\alpha, t)}{2}
$$

In order to highlight the potential of the proposed formulation, in the following of this section the non-stationary random excitation is modelled as a uniformly modulated white noise process. As known, such a model is successfully adopted in many engineering applications since it allows substantial simplifications in the mathematical formulation.

In this case, the stochastic process $F(t)$ in Eq.(33) is expressed multiplying a stationary Gaussian white noise process, $W(t)$, by an amplitude modulating deterministic function $\varphi(t)$, i.e. : $F(t)=\varphi(t) W(t)$. Accordingly, the autocorrelation function of $F(t)$ reads:

$$
R_{F F}\left(t_{1}, t_{2}\right)=2 \pi S_{W} \varphi\left(t_{1}\right) \varphi\left(t_{2}\right) \delta\left(t_{2}-t_{1}\right)
$$

where $S_{W}$ is the Power Spectral Density (PSD) of $W(t)$ and $\delta(t)$ denotes the Dirac delta function. By substituting Eq.(50) into the last of Eqs.(40) the following forcing vector is obtained:

$$
\mathbf{F}_{\mathbf{Y}}^{\mp}(\alpha, \mathrm{t}) \equiv \mathbf{F}_{\mathbf{Y}}(\alpha, \mathrm{t})=2 \pi \mathrm{S}_{W} \varphi^{2}(t)(\mathbf{V s}) \odot(\mathbf{V s})
$$

It follows that, when the input process is modelled as a uniformly modulated white noise, the proposed method involves much simpler mathematical derivations. 


\section{NUMERICAL APPLICATION}

In order to demonstrate the efficiency of the procedure presented in the previous section, a cantilever damaged steel beam studied in [1] subjected to an action $f(t)$ applied to the free-end is examined. Its geometry is reported in Fig.1.

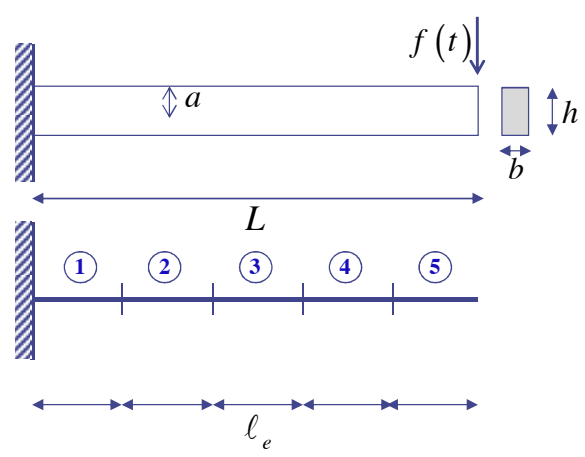

Figure 1: Geometry of the cantilever steel beam

The beam presents a rectangular cross-section with width $b=1 \mathrm{~mm}$ and height $h=7.8 \mathrm{~mm}$ and length $L=200 \mathrm{~mm}$. The Young's modulus and the material mass density are assumed $E=207000 \mathrm{~N} / \mathrm{mm}^{2}$ and $\rho=7860 \mathrm{Kg} / \mathrm{m}^{3}$, respectively. The cantilever beam has been modelled by $N_{\mathrm{e}}=5$ finite elements (see Fig.1), assuming positive upward vertical displacements and counter-clock wise rotations. Two different locations for the crack have been considered. The crack is supposed to be located in one case in the middle of the second element and in a second case study in the middle of the fourth element. Therefore the damaged element stiffness matrix is evaluated as $\mathbf{K}_{\mathrm{c}, 2}=\mathbf{T D}_{2}^{-1} \mathbf{T}^{T}$ or $\mathbf{K}_{\mathrm{c}, 4}=\mathbf{T} \mathbf{D}_{4}^{-1} \mathbf{T}^{T}$ (see Eq.12) respectively. The remaining four elements are modelled as undamaged beam finite elements.

The mean value $a_{0}$ of the uncertain-but-bounded crack depth $a$ defined as $a=a_{0}\left(1+\Delta \alpha \hat{e}_{\alpha}^{I}\right)$ is assumed to be $a_{0}=0.4 h$.

The different change into stiffness related to the location of the crack provides different values for the Rayleigh damping constants in Eq.(15). For the case of the crack located in the second element, the constants takes the values $c_{0}=83.927 \mathrm{~s}^{-1}$ and $c_{1}=0.0000138 \mathrm{~s}$, while for the case of damaged fourth element the constant assume the values $c_{0}=86.939 \mathrm{~s}^{-1}$ and $\mathrm{c}_{1}=0.0000139 \mathrm{~s}$, respectively. In both cases they are evaluated in such a way that the modal damping ratio for the first and second modes of the structure referred to the midpoint stiffness matrix $\mathbf{K}_{\mathrm{C}}$ is $\zeta_{0}=0.05$.

The analysis has been conducted for both cases of deterministic and stochastic action $f(t)$. Two different types of deterministic action have been considered: the first one represented by the unit step function $f(t)=U(t)$ while the second expressed by an harmonic force $f(t)=\sin (200 t)$.

Via the proposed procedure resumed in section 4.1 and applying Eqs.(31), the lower and upper bounds of the nodal displacement $u(t)$ of the free end of the cantilever beam, namely the 9-th nodal dynamic response, have been straightforwardly evaluated. The zero mean deviation has been assumed $\Delta \alpha=0.3$.

Figs.2a and $2 \mathrm{~b}$ show the time varying lower $\underline{u}_{9}(t)$ and upper $\bar{u}_{9}(t)$ bounds of the displacement (in mm) of the cantilever tip subjected to $U(t)$ considering the two crack locations, re- 
spectively in the elements 2 and 4 (as specified in the apex in brackets). In Fig.3 similar results are reported for the damaged beam under harmonic action.

Both bounds results coincide with the exact bounds in presence of crack with uncertainbut-bounded depth.

Comparing the response bounds trend it is worth to note that for both cases of acting load there is not correspondence among the peaks of the response which result shifted in time.

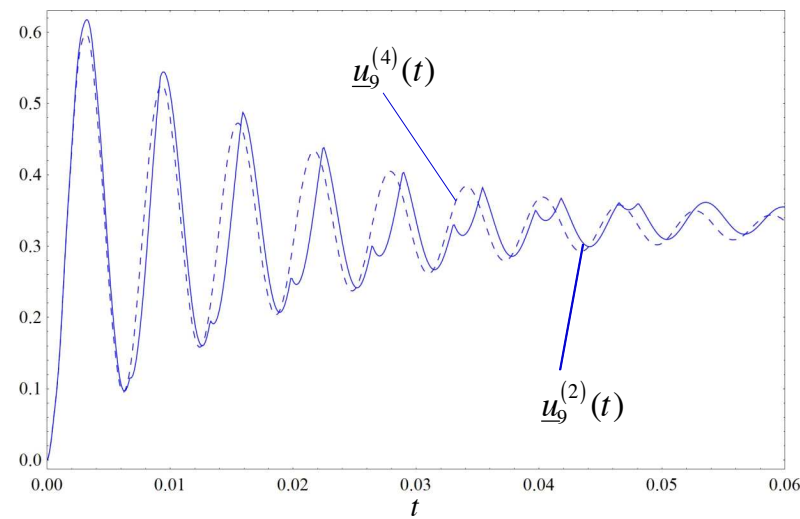

(a)

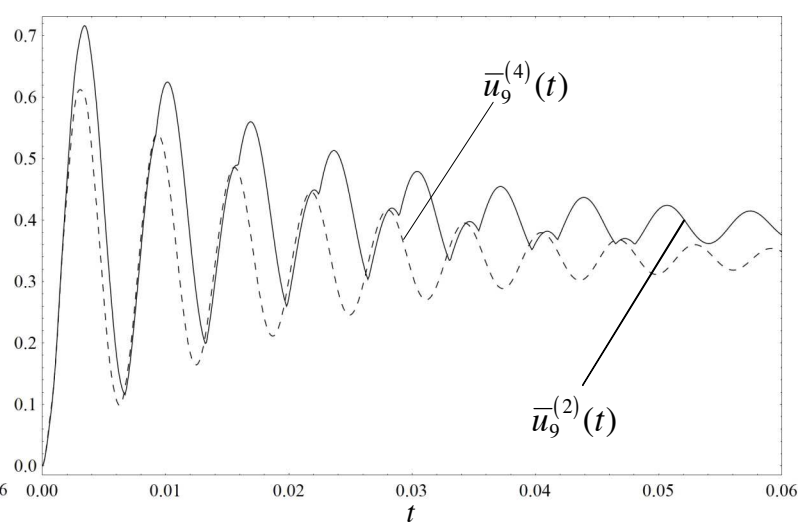

(b)

Figure 2: (a) Lower and (b) upper bound of the free-end displacement (in $\mathrm{mm}$ ) of the damaged cantilever beam with crack located at the middle of second element (continuous line) or fourth element (dashed line) under unit step action $U(t)$.

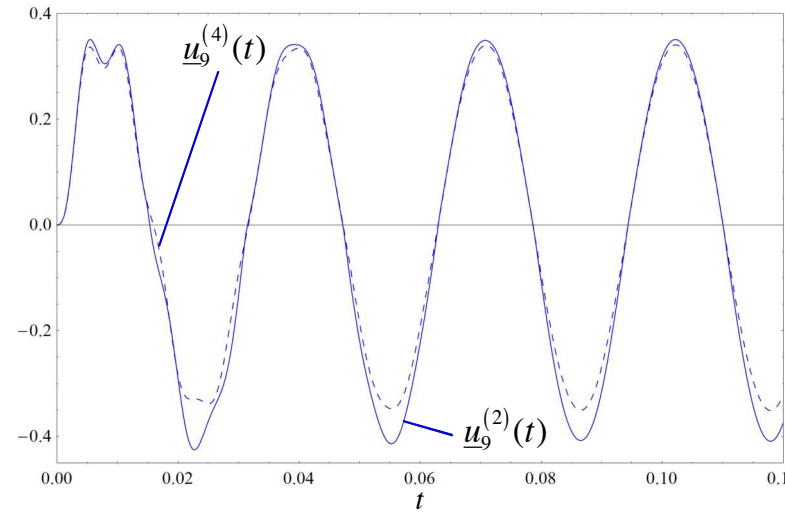

(a)

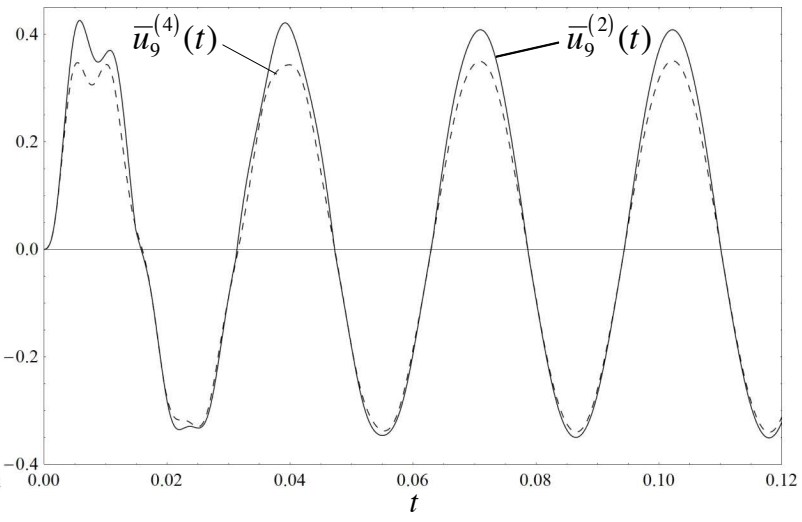

(b)

Figure 3: (a) Lower and (b) upper bound of the free-end displacement (in $\mathrm{mm}$ ) of the damaged cantilever beam with crack located at the middle of second element (continuous line) or fourth element (dashed line) under harmonic action.

The stochastic excitation acting at the free end of the beam has been defined as in Eq.(33) with $F(t)=\varphi(t) W(t)$. The stationary White Noise process has spectral density function $S_{W}=6 \times 10^{-4} \mathrm{~N}^{2}$ s and the modulating deterministic function $\varphi(t)$ has been expressed by $\varphi(t)=t(\exp (1) / \varepsilon) \exp (-t / \varepsilon)$ with $\varepsilon=0.3$.

Taking advantage of the mathematical simplifications, Eqs.(48) allow to evaluate the bounds of the nodal interval variance of the dynamic response in terms of displacement under uniformly modulated white noise.

In Figs.4a and 4b, as for the previous cases of deterministic actions, the bounds of the time varying displacement variances of the cantilever tip, $\underline{\sigma}_{U_{9}}^{2}(t)$ and $\bar{\sigma}_{U_{9}}^{2}(t)$ respectively, are reported and compared for the two crack locations considered (middle of second and fourth 
element). Also for this application the deviation amplitude is fixed $\Delta \alpha=0.3$. Same results are obtained performing the MonteCarlo simulation.

Figs. 4 show that for stochastic action the bounds peaks of the variance of the response in terms of free-end displacement are both lower when the crack is located at the middle of the fourth element respect to the case of crack located in the second element.

Moreover it is worth to note that the gap is larger between the upper bounds of the variances $\bar{\sigma}_{U_{9}^{(2)}}^{2}(t)$ and $\bar{\sigma}_{U_{9}^{(4)}}^{2}(t)$, respectively (see Fig.4b).
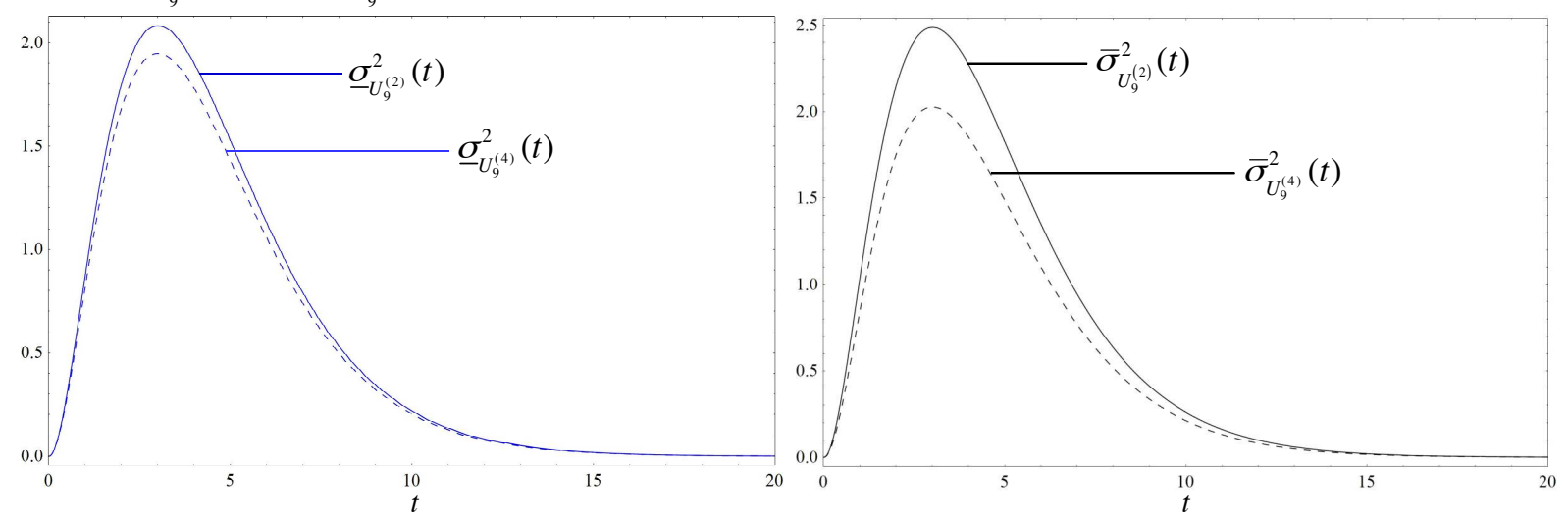

(a)

(b)

Figure 4: (a) Lower and (b) upper bound of the variance of the free-end displacement (in $\mathrm{mm}^{2}$ ) of the damaged cantilever beam with crack located at the middle of second element (continuous line) or fourth element (dashed line) under non-stationary random excitation.

Lastly in Figs.5 results in terms of bounds of the variances of the free-end displacement are reported for different deviation amplitude values, respectively $\Delta \alpha=0.3$ and $\Delta \alpha=0.1$.

Results shown in Fig.5a are referred to the case of crack located in the middle of the second element while those in Fig.5b are referred to the crack shifted in the fourth element.

Comparisons between the bounds of the variances of the free-end displacement show that larger intervals occur when the crack is located in the second element (see Fig.5a) while the intervals are narrow when the crack is located at the fourth element also for the larger deviation amplitude $\Delta \alpha=0.3$. It follows that the dynamic response of the damaged cantilever beam is more sensitive to the presence of the crack when the latter is located closer to the joint end, as expected.

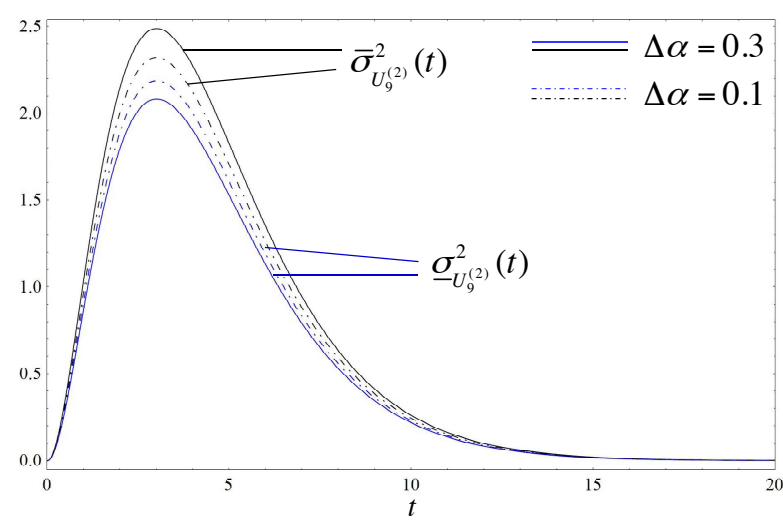

(a)

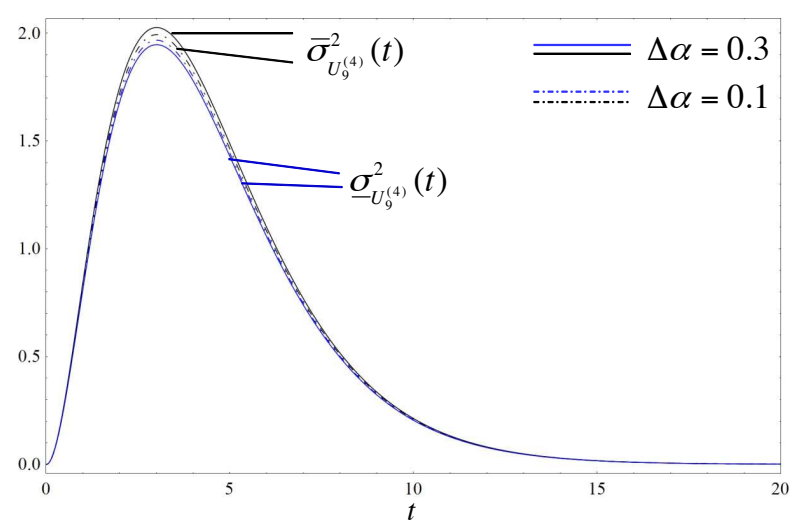

(b)

Figure 5: Lower and upper bounds of the variance of the free-end displacement (in $\mathrm{mm}^{2}$ ) of the damaged cantilever beam with crack located at the middle of (a) second element or (b) fourth element under non-stationary random excitation for deviation amplitude $\Delta \alpha=0.3$ (continuous line) and $\Delta \alpha=0.1$ (dot-dashed line). 


\section{CONCLUSIONS}

In this paper lower and upper bounds of the response of damaged beams with uncertainbut-bounded crack subjected to both deterministic and stochastic excitations modeled as zero mean Gaussian random processes are evaluated.

The proposed method allows to split both the time dependent deterministic and random response, in modal subspace, as sum of two aliquots: the midpoint or nominal solution and the deviation. For stochastic excitations, the differential equations governing the time-evolution of the midpoint and deviation covariance vectors of the response under zero-mean Gaussian stochastic input process are derived taking full advantage of the Kronecker algebra.

Main steps of the provided procedure summarize as follows: i) to define a finite element model of the beam in which the model of fully open crack is used to represent the damaged element; ii) to model the crack depth as an interval variable; iii) to evaluate in time domain the response for deterministic and stochastic excitation, by adopting an unified approach based on the Improved Interval Analysis via EUI.

Effectiveness and efficiency of the present method are shown in the numerical results, coinciding with the exact ones, concerning a cracked beam with uncertain-but-bounded stiffness properties under deterministic and uniformly modulated white noise excitation.

\section{REFERENCES}

[1] GL. Qian, SN. Gu, JS. Jiang. The dynamic behaviour and crack detection of a beam with a crack. Journal of Sound and Vibration, 138, 233-43, 1990.

[2] R. Ruotolo, C. Surace, P. Crespo, D. Storer. Harmonic analysis of the vibrations of a cantilevered beam with a closing crack. Computers \& Structures 6,1057-74, 1996.

[3] P. N. Saavedra, L.A. Cuitiño, Crack detection and vibration behavior of cracked beams. Computers \& Structures, 79, 1451-1459, 2001.

[4] P. Cacciola, N. Impollonia, G. Muscolino. Crack detection and location in a damaged beam vibrating under white noise. Computers \& Structures, 81,1773-1782, 2003.

[5] H. Nahvi, M. Jabbari, Crack detection in beams using experimental modal data and finite element model. International Journal of Mechanical Sciences, 47, 1477-1497, 2005.

[6] S. Teidj, A. Khamlichi, A.Driouach, Identification of Beam Cracks by Solution of an Inverse Problem. Procedia Technology, 22, 86-93, 2016.

[7] P. Cacciola, G. Muscolino, Dynamic response of a rectangular beam with a known nonpropagating crack of certain or uncertain depth, Computers \& Structures, 80, 2387-2396, 2002.

[8] R. E. Moore, Interval Analysis, Prentice-Hall, Englewood Cliffs, 1966.

[9] G. Muscolino, A. Sofi. Stochastic Response of Structures with Uncertain-But-Bounded Parameters via Improved Interval Analysis, Probabilistic Engineering Mechanics, 28, 152-163, 2012.

[10] R. Bellman. Introduction to matrix analysis, New York, McGraw-Hill, 1974.

[11] A. D'Aveni, G. Muscolino. Response of non-classically damped structures in the modal sunspace, Earthquake Engineering and Structural Dynamics, 24, 1267-1281, 1995. 